\title{
Superlattice structures of graphene-based armchair nanoribbons
}

\author{
H. Sevinçli, ${ }^{1}$ M. Topsakal, ${ }^{2}$ and S. Ciraci ${ }^{1,2, *}$ \\ ${ }^{1}$ Department of Physics, Bilkent University, Ankara 06800, Turkey \\ ${ }^{2}$ UNAM-Institute of Materials Science and Nanotechnology, Bilkent University, Ankara 06800, Turkey \\ (Received 27 July 2008; revised manuscript received 27 October 2008; published 1 December 2008)
}

\begin{abstract}
Based on first-principles calculations we predict that periodically repeated junctions of armchair graphene nanoribbons of different widths form multiple quantum well structures. In these superlattice heterostructures the width as well as the energy-band gap is modulated in real space and specific states are confined in certain segments. Not only the size modulation, but also composition modulation, such as periodically repeated and commensurate heterojunctions of boron nitride and graphene honeycomb nanoribbons, results in a multiple quantum well structure. The geometrical features of the constituent nanoribbons, namely, their widths and lengths, the form of the junction, as well as the symmetry of the resulting superlattice, are the structural parameters available to engineer electronic properties of these quantum structures. We present our analysis regarding the variation of the band gaps and the confined states with these structural parameters. Calculation of transmission coefficient through a double barrier resonant tunneling device formed from a finite segment of such a multiple quantum well structure and placed between metallic electrodes yields resonant peaks which can be identified with electronic states confined in the well. We show that these graphene-based quantum structures can introduce interesting concepts to design nanodevices. Relevance of the quantum structures are discussed in view of the most recent experimental results.
\end{abstract}

DOI: 10.1103/PhysRevB.78.245402

\section{INTRODUCTION}

Superlattices and various quantum structures obtained thereof have been the subject of active research in the past two decades. Commensurate or pseudomorphic junctions of two different semiconductors have been grown layer by layer to form a periodically repeating superlattice structure with sharp lattice-matched interface. ${ }^{1-3}$ Owing to the band discontinuities at the interface, they behave as a multiple quantum well structure according to the effective mass theory. Electrons confined in these quantum wells exhibit twodimensional (2D) electron-gas behavior with interesting quantum effects. ${ }^{4}$ Two-dimensional conduction-band electrons (valence-band holes) confined to the wells have displayed a number of electronic and optical properties. ${ }^{1-6}$ Formation of quantum structures in one dimensional (1D) systems have also been considered. For example, 1D multiple quantum wells or a series of quantum dots have been shown to form on a semiconducting zigzag nanotube by applying modulating radial strain and hence by forming bandgap modulation in real space. ${ }^{7,8}$ Multiple quantum wells are also obtained by the periodic junction of bare and hydrogenated zigzag carbon nanotubes. ${ }^{9}$ Similar quantum structures and confinement have been shown to occur in the junction of $(\mathrm{BN})_{n} \mathrm{C}_{n}$ linear chains. ${ }^{10}$

Recent advances in materials growth and control techniques have made the synthesis of the isolated 2D honeycomb crystal of graphene possible. ${ }^{11-13}$ Owing to its unusual electronic energy-band structure leading to the charge carriers resembling massless Dirac Fermions, graphene introduced new concepts and initiated active research. ${ }^{14-18}$ For example, quasi-1D graphene nanoribbons (GNRs) exhibit interesting size- and geometry-dependent electronic and magnetic properties. ${ }^{19-23}$ Also graphene nanoribbons less than $10 \mathrm{~nm}$ wide with ultrasmooth edges have already been produced. ${ }^{24}$ In the same work, a graphene structure having three segments of graphene nanoribbon with different widths
PACS number(s): 73.22.-f, 72.80.Rj, 75.70.Ak

has been obtained. This can be viewed as a finite segment of a graphene nanoribbon superlattice. Moreover, patterning of graphene nanoribbons ${ }^{13}$ and also graphene nanoribbons with varying widths ${ }^{17,25}$ has been achieved experimentally. With these developments the quantum well structures in graphenebased nanoribbons have been a subject of interest.

In this work, based on first-principles, as well as empirical tight-binding (ETB) calculations we show that periodically repeating heterojunctions made of armchair graphene nanoribbons of different widths can form stable superlattice structures. These superlattices are unique, since both their width and energy-band gap are modulated in real space. As a consequence, in addition to the propagating states, specific states are confined in certain regions. Confinement increases and turns to a complete localization when the lengths of the nanoribbon segments with different widths increase. This situation can be viewed as a series of quantum dots. Widths, lengths, chirality of constituent nanoribbons, and the symmetry of the junction can be varied to engineer these quantum structures with interesting electronic and transport properties. Further to these superlattices based on the width-modulated graphene armchair nanoribbons, we also show that multiple quantum well structures can be formed via composition modulation of commensurate heterojunction of boron nitride (BN) and graphene armchair nanoribbons of the same width. These quantum structures are of particular interest, since they show effects analogous to those of 2D semiconductor superlattices. Finally, our transport calculations demonstrate that specific finite-size quantum structures can operate as resonant tunneling double barriers (RTDB) with resonances-characteristics of confined quantum well states. In view of the recent experimental studies, ${ }^{13,17,24,25}$ present results related with graphene-based nanoribbons and their heterojunctions of diverse geometry can, in fact, be a candidate for a class of nanodevices with a richness of transport properties. 
Optimized atomic geometry of superlattices and corresponding electronic structure are obtained by performing first-principles plane-wave calculations ${ }^{26,27}$ within densityfunctional theory (DFT) (Refs. 28 and 29) using projector augmented wave (PAW) potentials. ${ }^{30}$ The exchangecorrelation potential is approximated by generalized gradient approximation (GGA) using PW91 functional ${ }^{31}$ both for spin-polarized and spin-unpolarized cases. All structures are treated within supercell geometry using periodic boundary conditions. A plane-wave basis set with kinetic-energy cutoff of $500 \mathrm{eV}$ is used for graphene as well as for BN. In the self-consistent potential and total-energy calculations the Brillouin zone $(\mathrm{BZ})$ is sampled by $(1 \times 1 \times 35)$ special $\mathbf{k}$ points for nanoribbons. This sampling is scaled according to the size of superlattices. All atomic positions and lattice constants are optimized by using the conjugate gradient method where total energy and atomic forces are minimized. The convergence for energy is chosen as $10^{-5} \mathrm{eV}$ between two steps, and the maximum force allowed on each atom is $0.02 \mathrm{eV} / \AA$. The superlattices having large width and hence unit cells with very large number of atoms are treated by using ETB calculations. The tight-binding Hamiltonian has the simple form $H_{\mathrm{ETB}}=-t \sum_{\langle i, j\rangle}\left(a_{i}^{\dagger} b_{j}+\right.$ H.c. $)$, where $a_{i}^{\dagger}\left(b_{j}^{\dagger}\right)$ creates an electron on site $\mathbf{R}_{i}\left(\mathbf{R}_{j}\right)$ of the $A(B)$ sublattice of the bipartite graphene lattice and $a_{i}\left(b_{j}\right)$ are the corresponding annihilation operators. The nearest-neighbor $(\langle i, j\rangle)$ hopping energy is $t \simeq 2.7 \mathrm{eV}$ (Ref. 32). In the transport calculations, we use nonequilibrium Green's function (NEGF) formalism ${ }^{33}$ implemented together with DFT (details of the $\mathrm{NEGF+DFT}$ method can be found in Ref. 34). As a requirement of the partitioning scheme we employ, a localizedorbital basis set (double $\zeta$ plus polarization numerical orbitals) is used in place of the plane-wave basis set. The results obtained from plane-wave and local-orbital DFT calculations are verified to yield consistent results. We further optimize the atomic structure before transport calculations. In transport calculations the exchange-correlation potential is approximated by GGA with PBE functional. ${ }^{35}$ During the selfconsistent calculation 21 special $\mathbf{k}$ points are used and the maximum energy difference is set to $10^{-4} \mathrm{eV}$ as the convergence criteria. In order to check the consistency of the different methods used, we compare the electronic band structures obtained from these calculations for $\operatorname{AGNR}(26)$ in Fig. 1.

\section{ARMCHAIR NANORIBBONS}

The electronic structure and the magnetic properties of GNRs are primarily determined by their edge shapes and widths. ${ }^{19-23}$ We first consider the bare and hydrogenterminated armchair graphene nanoribbons $\operatorname{AGNR}(n) ; n$ being the number of carbon atoms in the primitive unit cell. All $\operatorname{AGNR}(n)$ are semiconductors and their band gaps $E_{g}$ vary with $n$. We find that the band gap is small for $n=6 m-2(m$ being an integer), but from $n=6 m$ to $n=6 m+2$ it increases and passing through a maximum it becomes again small at the next minimum corresponding to $n=6 m+4$. As $E_{g}$ oscillates with $n$ its value decreases eventually to zero as $n \rightarrow \infty$. The results are in good agreement with the earlier works. ${ }^{23}$

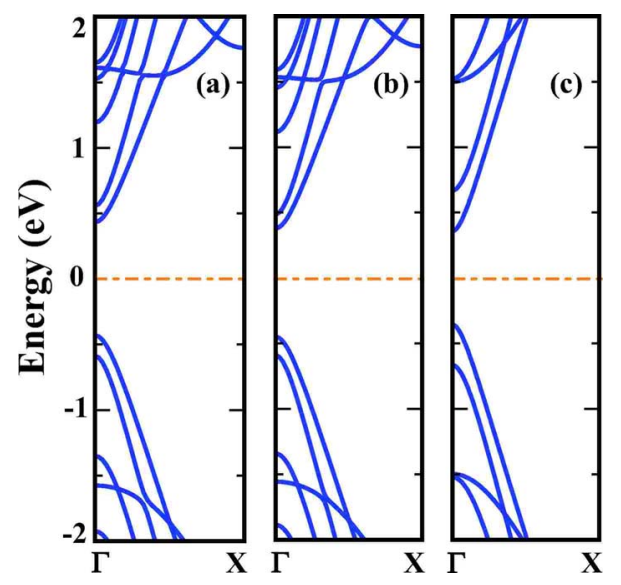

FIG. 1. (Color online) Band structure of $\operatorname{AGNR}(26)$ calculated with the three different methods used in this work: (a) plane-wave DFT, (b) localized-orbital DFT, (c) and ETB.

As an example, we present the electronic structure and charge density of AGNR(10) and AGNR(14) nanoribbons in Figs. 2(a) and 2(b). The band-gap values of bare AGNR(10) and AGNR(14) are calculated to be 0.44 and $1.10 \mathrm{eV}$, respectively. According to isosurface plots one distinguishes the relatively uniform states (having their charge uniformly distributed across the nanoribbon) from the edge states (having their charge accumulated at both edges of the ribbon). Owing to the interaction between two edges of the narrow ribbon, the bands of edge states split. Upon termination of the carbon dangling bonds by atomic hydrogen, the edge states of AGNR(10) and AGNR(14) disappear and their energy-band gaps change to 0.39 and $1.57 \mathrm{eV}$, respectively. Since hydrogen-terminated AGNRs are stable at ambient conditions, in the rest of the paper we consider their superlattices unless stated otherwise.

We note that defect-free bare and hydrogen-terminated armchair graphene nanoribbons have nonmagnetic ground
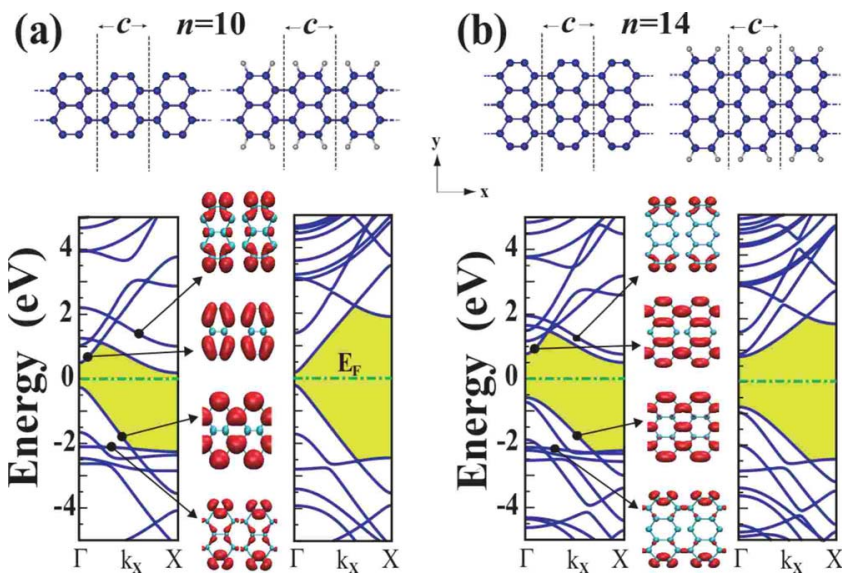

FIG. 2. (Color online) (a) Bare and hydrogen-terminated AGNR(10). Atomic geometry, electronic band structure, and isosurface charge densities of edge and "uniform" states. The primitive unit cell is delineated with dashed lines and includes $n=10$ carbon atoms. Carbon and hydrogen atoms are shown by large and small balls. (b) Same for AGNR(14). All data in this figure are calculated by first-principles method (Refs. 26 and 27). 
(a)

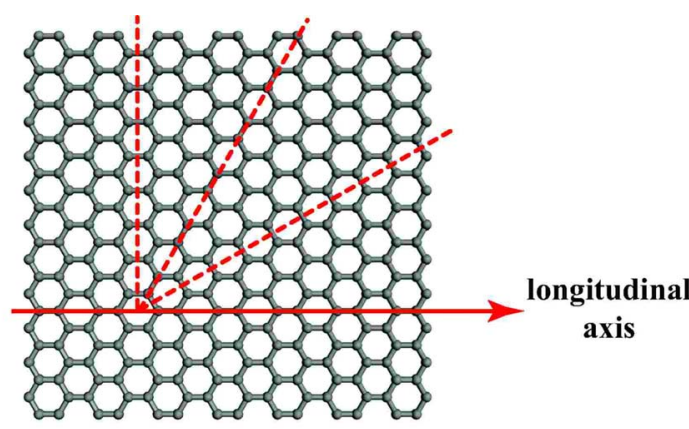

(b)

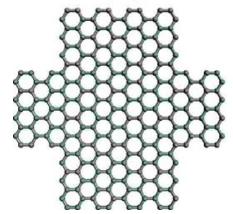

(c)

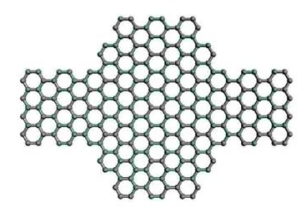

(d)

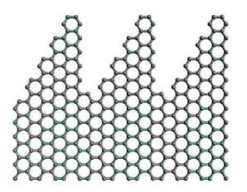

FIG. 3. (Color online) (a) Possible junction angles leading to armchair or zigzag edge shapes. $60^{\circ}$ angle with the longitudinal axis results in armchair edge at the interface, whereas $30^{\circ}$ and $90^{\circ}$ give zigzag edges. Some of the possible superlattice shapes are shown; namely, (b) sharp rectangular, (c) smooth, (d) and sawtoothlike.

state. Recently it has been shown that $3 d$-transition-metal atoms adsorbed to armchair nanoribbons induce permanent magnetic moment and hence give rise to interesting magnetic properties. ${ }^{36}$ Similarly, specific vacancy defects in AGNR can induce permanent magnetic moments. ${ }^{37}$ On the other hand, bare and hydrogen-terminated zigzag graphene nanoribbons (ZGNRs) have spin polarized edge states. ${ }^{20}$ The edge states having a high density of states near the Fermi energy give rise to a magnetic transition. Eventually ZGNR has edge magnetism with opposite spins populated at opposite edges. Here we consider only the superlattice structures made by armchair nanoribbons; the superlattice structures of zigzag nanoribbons are not discussed in this paper. In a short letter we showed that the spins can be confined in quantum structures formed from the zigzag ribbons. ${ }^{38}$

\section{SUPERLATTICE STRUCTURES}

\section{A. Graphene superlattices}

The remarkable properties of GNRs discussed above, especially their band gap rapidly varying with their widths, suggest that the heterostructures formed by the segments of AGNRs with different $n$ (or width) may have interesting functions. Here the crucial issues to be addressed are how the electronic structure will be affected from the discontinuity of crystal potential at the junction, what the character of the band discontinuity and the resulting band alignment will be, and whether these discontinuities will result in confined states. Relative to the longitudinal axis in an armchair direction one can distinguish three possible angles to make junctions with armchair or zigzag edge shapes as shown in Fig. 3(a). A $60^{\circ}$ angle gives rise to the armchair edge at the interface, whereas $30^{\circ}$ and $90^{\circ}$ angles result in zigzag edges. Some of the possible superlattice shapes are given in Figs. 3(b)-3(d).
AGSL (10,14;3,3)

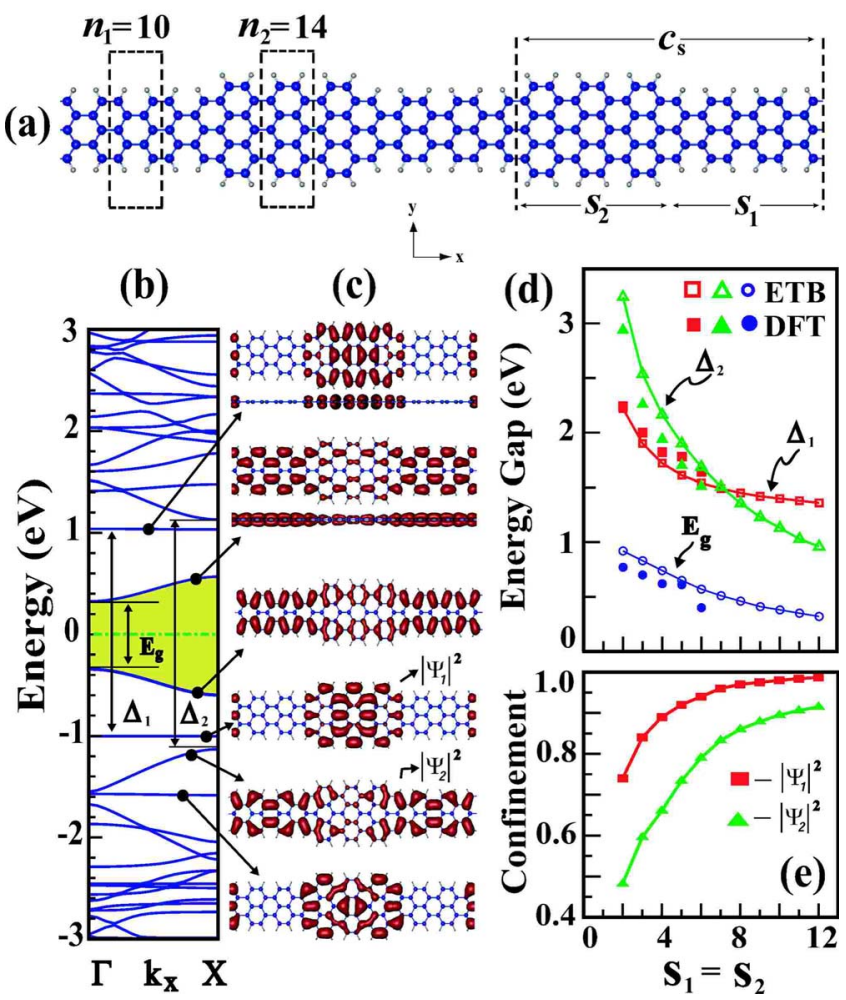

FIG. 4. (Color online) (a) Atomic structure of $\operatorname{AGSL}\left(n_{1}=10\right.$, $\left.n_{2}=14 ; s_{1}=3, s_{2}=3\right)$. The superlattice unit cell and primitive unit cell of each segment are delineated. (b) Band structure with flat bands corresponding to confined states. (c) Isosurface charge density of propagating and confined states. (d) Variation of various superlattice gaps with $s_{1}=s_{2}$. (e) Confinement of states versus $s_{1}$ $=s_{2}$ calculated by ETB. All data except those in (e) are calculated by using first-principles method (Refs. 26 and 27).

To avoid mixed edge shapes we focus on the junctions with smooth interfaces making $60^{\circ}$ angle with the longitudinal axis. To this end we first consider a superlattice $\operatorname{AGSL}\left(n_{1}, n_{2} ; s_{1}, s_{2}\right)$ made by the segments of $\operatorname{AGNR}\left(n_{1}\right)$ and $\operatorname{AGNR}\left(n_{2}\right)$. Here, $s_{1}$ and $s_{2}$ specify lengths (in terms of the number of unit cell) of the segments having different widths. Figure 4 shows the superlattice $\operatorname{AGSL}(10,14 ; 3,3)$. This superlattice is formed by periodically repeating the heterojunction of the segments of AGNR(10) and AGNR(14); each segment having three unit cells. The same superlattice can be visualized by periodically deleting one row of carbon atoms at both edges of $\mathrm{AGNR}(14)$ to form periodic $\operatorname{AGNR}(10) /$ AGNR(14) junction. Upon junction formation, dramatic changes occur in the band structure of this superlattice. While highest (lowest) valance (conduction) band is dispersive and their states propagate across the superlattice, the second valence and conduction bands are flat and their states are confined to the wider part of $\operatorname{AGSL}(10,14 ; 3,3)$ consisting of a segment of AGNR(14). These latter flat-band states are identified as confined states. Similar quantum-dot structures with sharp peaks in the density of states have also been revealed on the $Z$-shaped nanoribbons. ${ }^{39}$

The superlattice AGSL $(10,14 ; 3,3)$ in Fig. 4(a) can be viewed as a thin slab with periodically modulated width in 
the $x y$ plane. The electronic potential in this slab is lower $(V<0)$ than outside vacuum $(V=0)$. Normally, electrons in this thin slab propagate along the $x$ axis. Some electronic states in the wide segment are reflected from the discontinuities at the junction and eventually become confined. In these GNR superlattices the atomic arrangement and lattice constants at both sides of the junction are identical; the heterocharacter concerns only the different widths of the adjacent segments at different sides. According to this simple picture, the larger $\Delta n=n_{2}-n_{1}$, the stronger becomes the confinement. As justified in further discussions, these arguments are relevant for superlattices with long constituent segments. Note that AGNRs can be constructed by using two different unit cells (i.e., those consisting of complete hexagons or incomplete hexagons) reproducing the same crystal structure when translational symmetry is imposed [see the AGNR unit cells in Fig. 4(a)]. For the wider parts of the superlattice the one with complete hexagons is preferred in order to avoid having carbon atoms with coordination number 1 at the interfaces. For AGSL superlattices with reflection symmetry the narrow region is made by unit cells having complete or incomplete hexagons depending on whether $\left(n_{2}-n_{1}\right) / 4$ is an even or odd number, respectively.

In addition to $n_{1}, n_{2}, s_{1}, s_{2}$, the symmetry of the junction, $\Delta n=n_{2}-n_{1}$, even-odd disparity of $n_{1} / 2$ and $n_{2} / 2$, the type of the interface between two different ribbons, and the overall shape of the superlattice (namely, sharp rectangular, smooth wavy, or sawtoothlike) influence the electronic properties. We define the confinement as the weight of the wave function in the segment it becomes confined, say, $s_{2}$, i.e., $\int_{s_{2}}|\Psi(\mathbf{r})|^{2} d \mathbf{r}$, and examine the variation of specific band gaps and confinement of states with the size of the segments and supercell. For example, as shown in Fig. 4(d), as $s_{1}=s_{2}$ increases, various superlattice band gaps decrease, but the confinement of specific states increases. This is a clear evidence for quantum size effect. The role of $s_{1}$ and $s_{2}$ is, however, different in the confinement of a state. For example, for $\Psi_{1}(\mathbf{r})$ the effect of barrier is complete already for $s_{1} \geq s_{2}$, and the confinement of the state does not change considerably when $s_{2}$ is fixed but $s_{1}$ is increased. On the other hand, the confinement of this state increases when $s_{1}$ is fixed, but $s_{2}$ increased. Other confined states display similar behavior. It appears that as a consequence of quantum size effect the energy of $\Psi_{1}(\mathbf{r})$ decreases as the size $s_{2}$ of the well increases. Less kinetic energy of the confined electron (i.e., hence higher barrier height) causes suppression of the penetration into the barrier region. When $s_{2}$ is kept fixed, the energy of the confined electron also remains the same. The exponential decay rate of the tail is unchanged under the barrier region, only the length of the tail changes with $s_{1}$. Consequently, the strength of confinement is less sensitive to $s_{1}$ once the electron is confined to the $s_{2}$ region.

The rate of change of band gaps with strain $\epsilon=\Delta c / c(\Delta c$ being the change of lattice constant under uniaxial stress along the $x$ axis.) of nanoribbon superlattice structures is also a notable feature, which may be interesting for future straingauge device applications. In Figs. 5(a) and 5(b) we present our results for the variation of $E_{g}$ and other relevant gaps of the nanoribbon superlattice $\operatorname{AGSL}(10,14 ; 3,3)$ and also its constituent AGNRs for the sake of comparison. Strong de-

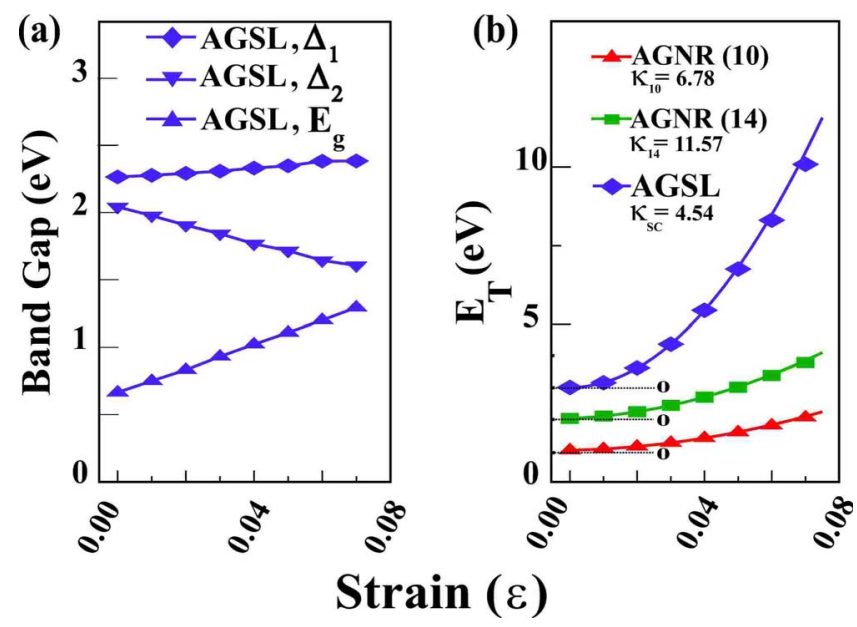

FIG. 5. (Color online) (a) Variation of band gaps $E_{g}, \Delta_{1}$, and $\Delta_{2}$ of the nanoribbon superlattice AGSL(10,14:3,3). (b) Variation of the total energy $E_{T}$ with respect to $\epsilon$ and the force constant $\kappa_{\mathrm{SL}}$ [in $\mathrm{eV} / \AA]$ for $\operatorname{AGSL}(10,14 ; 3,3)$ and its constituent nanoribbons. All data in this figure are calculated by using first-principles method (Refs. 26 and 27).

pendence on $\epsilon$ is impressive. That these superlattice structures are quasi-1D stiff materials is shown by the variations of their total energies $E_{T}$ with respect to $\epsilon$ in Fig. 5(b). The force constant of the superlattice calculated by $\kappa_{\mathrm{SL}}$ $=\partial^{2} E_{T} / \partial c^{2}$ is in good agreement with the equivalent force constant $\kappa_{\text {eq,SL }}=\left(s_{1} / \kappa_{n_{1}}+s_{2} / \kappa_{n_{2}}\right)^{-1}$ calculated in terms of the force constants of the constituent nanoribbons $\kappa_{n_{1}}$ and $\kappa_{n_{2}}$. This shows that elastic properties of superlattices comply with Hooke's law for small $\Delta n=n_{2}-n_{1}$.

Deviations of $s_{1}$ from $s_{2}$ causes $E_{g}$ and the band structure to change (see Fig. 6). For example, $\operatorname{AGSL}\left(10,14 ; s_{1}, s_{2}\right)$ has $E_{g}=0.66,0.48,0.38,0.32,0.27,0.22 \mathrm{eV}$ for $s_{2}=3$, but $s_{1}$ $=3,4,5,6,7,8, \quad$ respectively. Conversely, $E_{g}$ $=0.66,0.72,079,0.83,0.84,0.86 \mathrm{eV}$ for $s_{1}=3$, but $s_{2}$ $=3,4,5,6,7,8$, respectively. On the other hand, the energy of the flat-band states $\Delta_{1}$ confined to $s_{2}$ and their weight are practically independent of $s_{1}$.

Variation of $\Delta n=n_{1}-n_{2}$ results in a wide variety of electronic structures. For example, in contrast to AGSL $(10,14 ; 3,3)$ the highest valence and lowest conduction bands of AGSL $(10,18 ; 3,3)$ are flat bands with $E_{g}=0.70 \mathrm{eV}$; dispersive bands occur as second valence and conduction bands having a gap of $1.19 \mathrm{eV}$ between them (see Fig. 7). Here $\Delta_{2}=1.18,0.86,0.66,0.51,0.43,0.35 \mathrm{eV}$ with $s_{2}=3$ but $s_{1}=3,4,5,6,7,8$, respectively, whereas $\Delta_{1}$ remains unaltered $\left(\Delta_{1} \approx 0.70 \mathrm{eV}\right)$. The situation is even more complex for AGSL(10,22;3,3). While the first and the second bands are dispersive in both valence and conduction bands with a direct band gap $0.23 \mathrm{eV}$, flat bands occur as third bands with a gap of $2.61 \mathrm{eV}$ between them. The states associated with the flat bands near the Fermi level are confined at the wider part of the superlattice. It should be noted that the band gap of nanoribbons $E_{g}$ is underestimated by the DFT calculations. ${ }^{40}$ Since we consider structures which have already a band gap, its actual value does not affect our discussion qualitatively, but an enhancement of predicted properties (such as the strength of confinement) can be expected. 
(a)
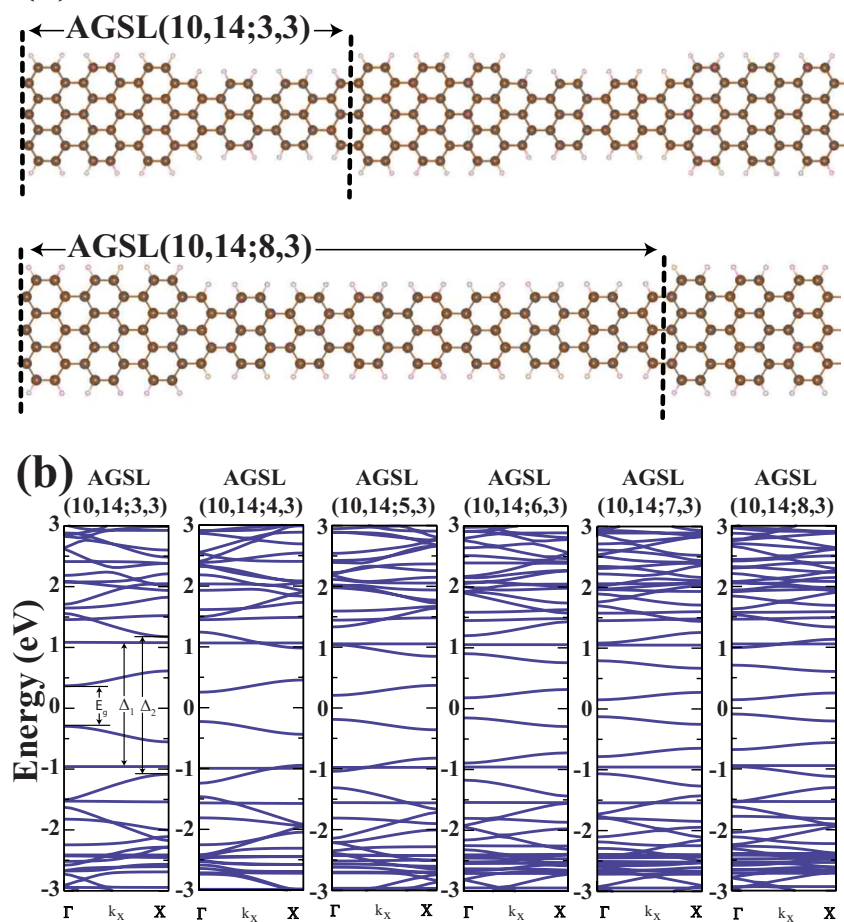

(c)

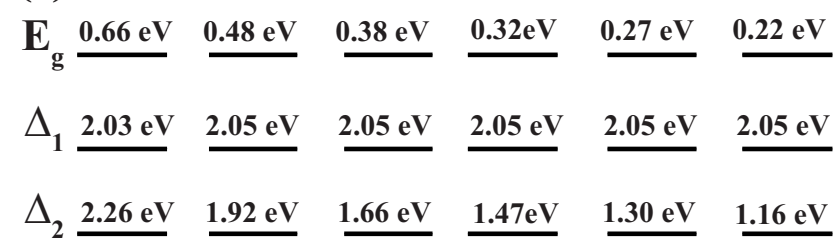

FIG. 6. (Color online) The effect of the variation of narrower region $s_{1}$ of AGSL $\left(10,14 ; s_{1}, 3\right)$ from $s_{1}=3$ to 8 . (a) Atomic structure and superlattice unit cell. (b) The variation of band structures. (c) The numerical values for energy gaps $E_{g}, \Delta_{1}$, and $\Delta_{2}$. $E_{g}$ is the actual band gap of the structure which comes from a dispersive state. $\Delta_{1}$ is the band gap of highest localized state while $\Delta_{2}$ is the band gap for the next dispersive state. The energy of the flat-band states related with $\Delta_{1}$ are confined to $s_{2}$ and their weights are practically independent of $s_{1}$. All data in this figure are calculated by using first-principles method (Refs. 26 and 27).

Moreover in Fig. 8 we demonstrate that the confined states can occur not only in narrow (small $n_{1}$ and $n_{2}$ ), but also in wide superlattices having significant modulation of the width. The above trends corresponding to small $n_{1}$ and $n_{2}$ become even more interesting when $n_{1}$ and $n_{2}$ increase. The electronic band structure of $\operatorname{AGSL}\left(n_{1}, n_{2} ; s_{1}, s_{2}\right)$ with $n_{1}$ $=42$ or 82 , but $n_{2}>n_{1}$ and $s_{1}=s_{2} \geq 3$ calculated using ETB method shows that for small $\Delta n$, confinement is weak and bands are dispersive, but confinement increases as $\Delta n$ increases. Interestingly, $E_{g}$ of $\operatorname{AGSL}\left(n_{1}=42, n_{2} ; 3,3\right)$ is, respectively, $0.46,0.12,0.49$, and $0.04 \mathrm{eV}$ for $n_{2}=46,50,54$, and 58. In ETB method used here, the Bloch states having band index $l$ and wave vector $\mathbf{k}$ are expressed in terms of the linear combination of the orthonormalized Bloch sums $\chi_{i}(\mathbf{k}, \mathbf{r})$ constructed for each atomic orbital $p_{z}$ localized at different carbon atoms, $i$, with the proper phase of $\mathbf{k}$, namely,
AGSL (10,18;3,3)

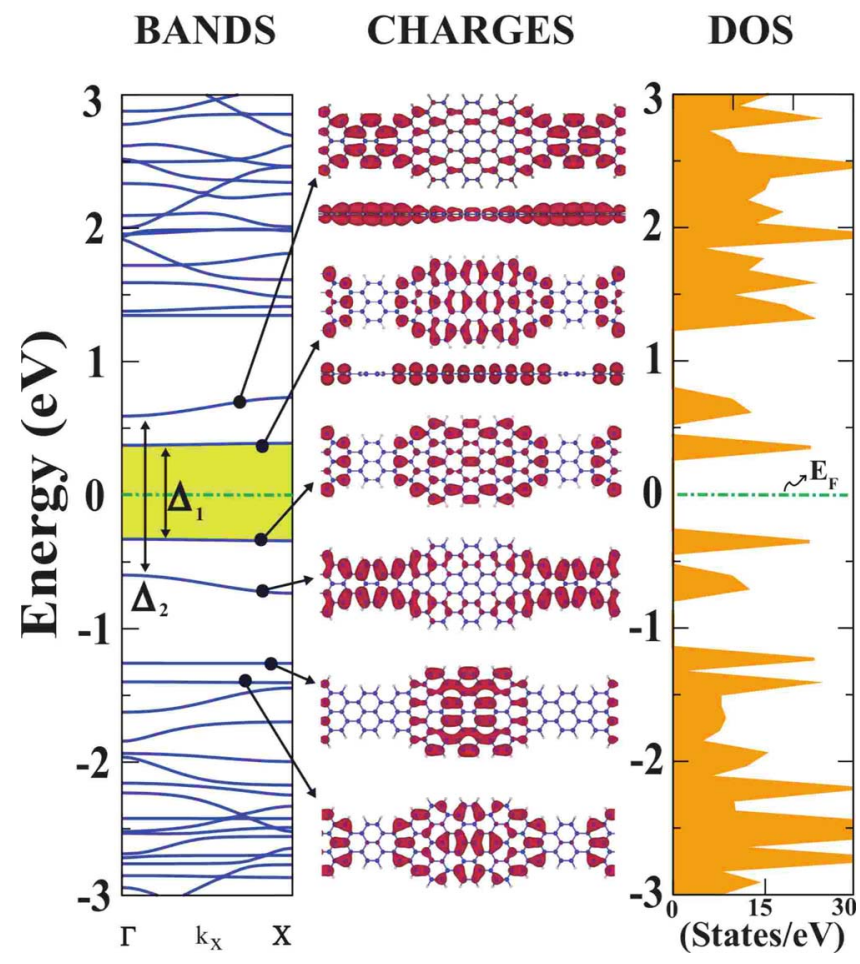

FIG. 7. (Color online) (a) Band structure of $\operatorname{AGSL}(10,18 ; 3,3)$ with flat bands corresponding to confined states $\left(\Delta_{1}=E_{g}\right)$. (b) Isosurface charge density of propagating and confined states. (c) Density of states (DOS) of AGSL $(10,18 ; 3,3)$ with sharp peaks corresponding to confined states. Calculations are performed by using first-principles method (Refs. 26 and 27).

$\psi_{l}(\mathbf{k}, \mathbf{r})=\sum_{i} a_{i, l}(\mathbf{k}) \chi_{i}(\mathbf{k}, \mathbf{r})$. Accordingly, the contribution of the orbital at site $i$ to the normalized charge density of $\psi_{l}(\mathbf{k}, \mathbf{r})$ is given by $\rho_{i}=\Sigma_{l}\left|a_{i, l}(\mathbf{k})\right|^{2}$. In Fig. $8, \rho_{i}$ is scaled with the radius of circles located at atomic site $i$.

Electronic structure is also strongly dependent on whether the geometry of the superlattice is symmetric (having a reflection symmetry with respect to the superlattice axis along the $x$ direction) or saddle (one side is straight, other side is periodically carved), all having the same $\Delta n$. While the saddle structure of $\operatorname{AGSL}(10,18 ; 3,3)$ has largest direct gap between dispersive conduction and valence bands, its symmetric structure has smallest gap, but largest number of confined states. Hornlike smooth connection between wide and narrow segments (where the ribbon is carved from both sides smoothly and symmetrically) may give rise to adiabatic electron transport and focused electron emission. ${ }^{41}$

\section{B. BN-graphene superlattice}

Recently, two-dimensional BN honeycomb structure has been synthesized. ${ }^{42}$ While several features of $\mathrm{BN}$ honeycomb structure including its lattice constant are similar to those of graphene, the $s p^{2}$ bonding gains an ionic character due to different electronegativity of $\mathrm{B}$ and $\mathrm{N}$ atoms in $\mathrm{BN}$. In contrast to two linear bands crossing at the $\mathrm{K}$ and $\mathrm{K}^{\prime}$ points of 


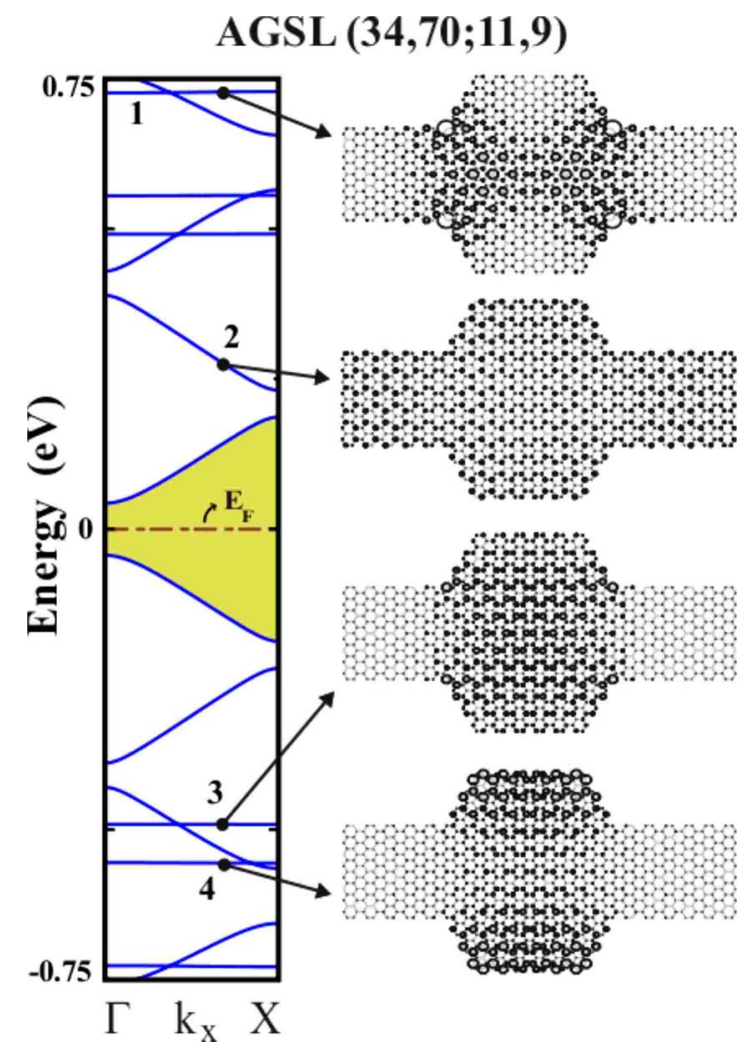

FIG. 8. (Color online) Energy-band structure of the AGSL $(34,70 ; 11,9)$ superlattice and the charge densities of selected bands. As seen clearly, states associated with flat bands 1, 3, and 4 are confined but the state with dispersive band indicated by 2 is propagating. Calculations have been performed using ETB method.

$\mathrm{BZ}$ of semimetallic graphene, $\mathrm{BN}$ honeycomb structure is a semiconductor due to a large gap between conduction and valence bands. Not only $2 \mathrm{D} \mathrm{BN}$, but also its ribbons are semiconductor with large band gap. ${ }^{43}$ While the band gap of GNR being strongly dependent on its width, the band gap of BN nanoribbons does not change significantly with width, especially for large $n .{ }^{44}$ Hence, graphene and BN nanoribbons, both having commensurate structure and being semiconductor with different band gaps, can make ideal materials for a multiple quantum well structure. In fact, this situation is reminiscent of the GaAs/AlAs 2D semiconductor superlattices studied actively during last two decades. ${ }^{2}$ Our arguments are clarified in Fig. 9, where a 1D analog of the 2D semiconductor superlattices through compositional modulated nanoribbons is presented. BN and graphene nanoribbons of the same width are lattice matched and can form superlattices with multiple quantum well structure having confined states. We consider a periodic junction of the segment of armchair BN nanoribbon with $n_{1}=18$ and $s_{1}=3$ to the segment of armchair GNR with $n_{2}=18$ and $s_{2}=3$ to form a superlattice structure. While periodic $\mathrm{BN}$ and graphene nanoribbons by themselves have band gaps of $\sim 5$ and $0.8 \mathrm{eV}$, respectively, the band gap of $\mathrm{BN}(18) / \mathrm{AGNR}(18)$ is only 0.8 $\mathrm{eV}$ indicating a normal (type I) band alignment. ${ }^{2}$ Under these circumstances, a state propagating in one segment becomes confined if its energy falls in the band gap of the adjacent BN segment. In Fig. 9(b), the dispersive minibands and nondis-

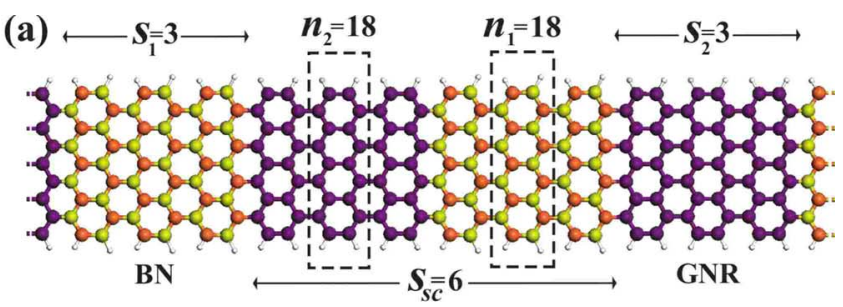

(b)

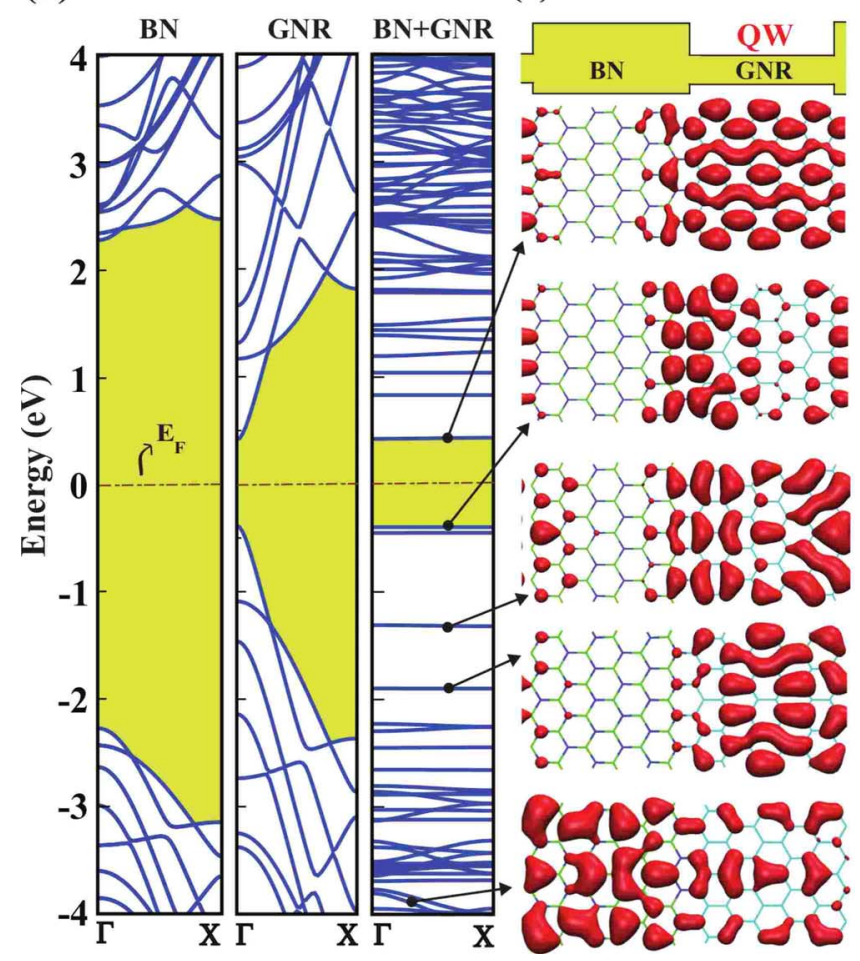

FIG. 9. (Color online) One-dimensional superlattice structure formed from the junction of $\mathrm{BN}$ and graphene armchair nanoribbons. (a) Atomic structure and superlattice parameters. (b) Band structures of constituent $\mathrm{BN}$ and graphene armchair nanoribbons having 18 atoms in their unit cells and the band structure of the superlattice $\mathrm{BN}(18) / \mathrm{AGNR}(18)$ each segment having three unit cells $\left(s_{1}=s_{2}=3\right)$. (c) Energy-band diagram in real space forming multiple quantum wells (QW) in graphene segments (zones). Isosurface charge densities of states confined to QWs and propagating states are presented for selected bands. All data in this figure are calculated by first-principles method (Refs. 26 and 27).

persive quantum well states are clearly seen. That these quantum well states are confined in the graphene zone (which has small band gap as compared to the band gap of $\mathrm{BN}$ ribbon) are demonstrated by isosurface plots of charge densities in Fig. 9(c). In contrast to the confined states, the propagating states have charge densities in both graphene and $\mathrm{BN}$ zones of the superlattice. This is another class of heterostructure where electronic potentials are different at both sides of junction. Its function can even be advanced by implementing the size modulation in addition to the compositional one.

\section{RTDB device}

Finally we focus on possible application of armchair graphene-based heterostructures and investigate the transport 
(a)
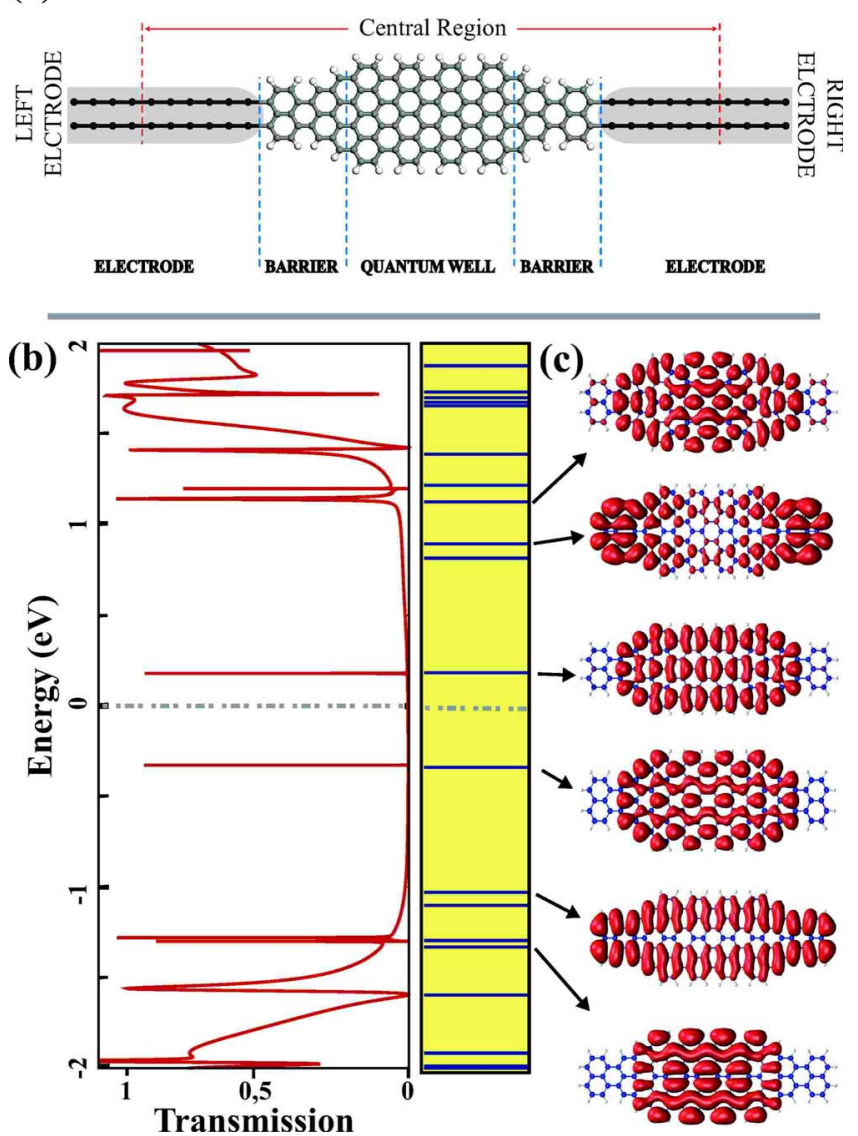

FIG. 10. (Color online) (a) Atomic structure of a RTDB device made from a symmetric segment of AGNR(10)/AGNR(18) system and connected to two metallic electrodes. (b) Transmission coefficient $\boldsymbol{T}$ versus energy under zero bias calculated using nonequilibrium Green's function approach using local-orbital DFT. (c) The energy-level diagram and the charge densities of selected confined and extended states of the decoupled device calculated using planewave DFT.

properties of a finite segment of AGSL. In accordance with the results in Sec. III A, we examine the effects of confinement on the transport properties upon modulation of the width of ribbons. We consider a finite ribbon with AGNR(10) and AGNR(18) as constituent parts each having total length of 8 AGNR unit cells as shown in Fig. 10. Such a system is relevant for applications and uses lowest unoccupied molecular orbital (LUMO) and highest occupied molecular orbital (HOMO) states in the confined region. ${ }^{3}$ For the sake of illustration we consider fictitious metallic electrodes of two widely separated (weakly coupled) carbon chains ${ }^{45}$ altogether having four quantum conductance channels. As long as the tunneling resonances are considered these electrodes are able to reproduce qualitatively the same transmission spectrum, e.g., with two-dimensional graphene. Six principal layers of electrodes are included at both sides of the RTDB as parts of the central region. Metallic electrodes make perfect contacts with the ribbon.
The transmission spectrum, $\boldsymbol{T}(E)$, calculated selfconsistently using nonequilibrium Green's function method ${ }^{34}$ reflects the combined electronic structure of the central RTDB device, electrodes, and their contacts. Figure 10 presents the calculated transmission curve. Confined LUMO and HOMO states and other confined states identified through the energy-level diagram and isosurface chargedensity plots give rise to sharp peaks originating from resonant tunneling effect. States extending to the entire ribbon are coupled with the states of the electrode. These states are shifted and contributed to broad structures in the transmission spectrum. We also note that the resonant tunneling effect is not affected by the width of the barrier regions [namely, narrow AGNR(10) segments in Fig. 10(a)], considerably. The confined LUMO and HOMO states are weakly sensitive to the length of the barriers. This feature of confined states can also be observed from the energies of confined states (flat bands) of Fig. 6. For tunneling transport, wider barriers mean exponentially lower coupling between the quantum well region and the electrodes, which result in even sharper peaks at approximately the same energies.

It is shown experimentally that transport through GNRs is primarily influenced by the boundary shape. Also charge confinement influences the conductance significantly. ${ }^{13,24,25}$ Özyilmaz et al. ${ }^{25}$ reported suppression of conductance of graphene nanoribbons by Coulomb blockade due to formation of multiple quantum dots in series which are formed during the etching process. Even though these quantum wells were not produced in a controlled manner, the results in transport measurements support our calculations of resonant tunneling effect. Geometrical confinement and multiple quantum wells can also be realized from 2D graphene by using side gates. ${ }^{46}$ Recently, the fabrication of graphene nanoribbon field effect transistor has been reported. ${ }^{47}$ In summary, these works provide evidences that the superlattice structures of graphene nanoribbons are relevant experimentally.

\section{CONCLUSIONS}

In this paper, various types of quantum structures are revealed through size and composition modulations of graphene-based armchair nanoribbons having honeycomb structure. The confinement of electron and hole states are shown to lead to interesting effects for new device applications based on graphene nanoribbons. A rich variety of electronic structure and resulting transport properties can be obtained by varying the structural parameters of superlattices. Variation of the band gaps, confined state energies, and the strength of confinement with the width and length and composition of the constituent nanoribbons are the key features for future applications.

\section{ACKNOWLEDGMENTS}

We acknowledge fruitful discussions with E. Durgun. Part of the computations have been carried out by using UYBHM at Istanbul Technical University through Grant No. 2-0242007. 
*ciraci@fen.bilkent.edu.tr

${ }^{1}$ L. Esaki and R. Tsu, IBM J. Res. Dev. 14, 61 (1970).

${ }^{2}$ L. Esaki and L. L. Chang, Phys. Rev. Lett. 33, 495 (1974); L. Esaki, Highlights in Condensed Matter Physics and Future Prospects, NATO Advanced Studies Institute, Series B: Physics (Plenum, New York, 1991), p. 285, several relevant references can be found in this proceedings.

${ }^{3}$ L. L. Chang, L. Esaki, and R. Tsu, Appl. Phys. Lett. 24, 593 (1974).

${ }^{4} \mathrm{G}$. Bastard, Wave Mechanics Applied to Semiconductor Heterostructures (Les Editions de Physique, Les Ullis, France, 1988).

${ }^{5}$ S. Ciraci and I. P. Batra, Phys. Rev. Lett. 58, 2114 (1987).

${ }^{6}$ S. Ciraci and I. P. Batra, Phys. Rev. B 36, 1225 (1987); 38, 1835 (1988); S. Ciraci, A. Baratoff, and I. P. Batra, ibid. 41, 2763 (1990).

${ }^{7}$ C. Kilic, S. Ciraci, O. Gulseren, and T. Yildirim, Phys. Rev. B 62, R16345 (2000).

${ }^{8}$ O. Gulseren, T. Yildirim, S. Ciraci, and C. Kilic, Phys. Rev. B 65, 155410 (2002).

${ }^{9}$ O. Gulseren, T. Yildirim, and S. Ciraci, Phys. Rev. B 68, 115419 (2003).

${ }^{10}$ S. Tongay, S. Dag, E. Durgun, R. T. Senger, and S. Ciraci, J. Phys.: Condens. Matter 17, 3823 (2005).

${ }^{11}$ K. S. Novoselov, A. K. Geim, S. V. Morozov, D. Jiang, Y. Zhang, S. V. Dubonos, I. V. Grigorieva, and A. A. Firsov, Science 306, 666 (2004).

${ }^{12}$ Y. Zhang, Y.-W. Tan, H. L. Stormer, and P. Kim, Nature (London) 438, 201 (2005).

${ }^{13}$ C. Berger, Z. Song, X. Li, X. Wu, N. Brown, C. Naud, D. Mayou, T. Li, J. Hass, A. N. Marchenkov, E. H. Conrad, P. N. First, and W. A. de Heer, Science 312, 1191 (2006).

${ }^{14}$ M. I. Katsnelson, K. S. Novoselov, and A. K. Geim, Nat. Phys. 2, 620 (2006)

${ }^{15}$ K. S. Novoselov, A. K. Geim, S. V. Morozov, D. Jiang, M. I. Katsnelson, I. V. Grigorieva, S. V. Dubonos, and A. A. Firsov, Nature (London) 438, 197 (2005).

${ }^{16}$ A. K. Geim and K. S. Novoselov, Nature Mater. 6, 183 (2007).

${ }^{17}$ M. Y. Han, B. Özyilmaz, Y. Zhang, and P. Kim, Phys. Rev. Lett. 98, 206805 (2007).

${ }^{18}$ Y.-W. Son, M. L. Cohen, and S. G. Louie, Nature (London) 444, 347 (2006).

${ }^{19}$ K. Nakada, M. Fujita, G. Dresselhaus, and M. S. Dresselhaus, Phys. Rev. B 54, 17954 (1996)

${ }^{20}$ M. Fujita, K. Wakabayashi, K. Nakada, and K. Kusakabe, J. Phys. Soc. Jpn. 65, 1920 (1996).

${ }^{21}$ V. Barone, O. Hod, and G. E. Scuseria, Nano Lett. 6, 2748 (2006).
${ }^{22}$ E. Rudberg, P. Saek, and Y. Luoi, Nano Lett. 7, 2211 (2007).

${ }^{23}$ Y.-W. Son, M. L. Cohen, and S. G. Louie, Phys. Rev. Lett. 97, 216803 (2006).

${ }^{24}$ X. Li, L. Zhang, S. Lee, and H. Dai, Science 319, 1229 (2008).

${ }^{25}$ B. Özyilmaz, P. J. Herrero, D. Efetov, and P. Kim, Appl. Phys. Lett. 91, 192107 (2007).

${ }^{26}$ G. Kresse and J. Hafner, Phys. Rev. B 47, 558 (1993).

${ }^{27}$ G. Kresse and J. Furthmüller, Phys. Rev. B 54, 11169 (1996).

${ }^{28}$ W. Kohn and L. J. Sham, Phys. Rev. 140, A1133 (1965).

${ }^{29}$ P. Hohenberg and W. Kohn, Phys. Rev. 136, B864 (1964).

${ }^{30}$ P. E. Blöchl, Phys. Rev. B 50, 17953 (1994).

${ }^{31}$ J. P. Perdew, J. A. Chevary, S. H. Vosko, K. A. Jackson, M. R. Pederson, D. J. Singh, and C. Fiolhais, Phys. Rev. B 46, 6671 (1992).

${ }^{32}$ C. H. Xu, C. Z. Wang, C. T. Chan, and K. M. Ho, J. Phys.: Condens. Matter 4, 6047 (1992).

${ }^{33}$ S. Datta, Electronic Transport in Mesoscopic Systems (Cambridge University Press, Cambridge, 1995)

${ }^{34}$ M. Brandbyge, J.-L. Mozos, P. Ordejon, J. Taylor, and K. Stokbro, Phys. Rev. B 65, 165401 (2002).

${ }^{35}$ J. P. Perdew, K. Burke, and M. Ernzerhof, Phys. Rev. Lett. 77, 3865 (1996).

${ }^{36}$ H. Sevinçli, M. Topsakal, E. Durgun, and S. Ciraci, Phys. Rev. B 77, 195434 (2008).

${ }^{37}$ M. Topsakal, E. Akturk, H. Sevincli, and S. Ciraci, arXiv:0808.1468 (unpublished).

${ }^{38}$ M. Topsakal, H. Sevinçli, and S. Ciraci, Appl. Phys. Lett. 92 173118 (2008).

${ }^{39}$ Z. F. Wang, Q. W. Shi, Q. Li, X. Wang, and J. G. Hou, Appl. Phys. Lett. 91, 053109 (2007).

${ }^{40}$ L. Yang, C.-H. Park, Y.-W. Son, M. L. Cohen, and S. G. Louie, Phys. Rev. Lett. 99, 186801 (2007).

${ }^{41}$ E. Tekman and S. Ciraci, Phys. Rev. B 40, 8559 (1989); S. Ciraci and I. P. Batra, ibid. 36, 6194 (1987).

${ }^{42}$ K. S. Novoselov, D. Jiang, F. Schedin, V. V. Khotkevich, S. V. Morozo, and A. K. Geim, Proc. Natl. Acad. Sci. U.S.A. 102, 10451 (2005).

${ }^{43}$ C.-H. Park and S. G. Louie, Nano Lett. 8, 2200 (2008).

${ }^{44}$ M. Topsakal, E. Akturk, C. Ataca, and S. Ciraci (unpublished).

${ }^{45}$ S. Tongay, R. T. Senger, S. Dag, and S. Ciraci, Phys. Rev. Lett. 93, 136404 (2004).

${ }^{46}$ L. A. Ponomarenko, F. Schedin, M. I. Katsnelson, R. Yang, E. W. Hill, K. S. Novoselov, and A. K. Geim, Science 320, 356 (2008).

${ }^{47}$ X. Wang, Y. Ouyang, X. Li, H. Wang, J. Guo, and H. Dai, Phys. Rev. Lett. 100, 206803 (2008). 